\title{
Online Collections of Vietnam War-Era U.S. Topographic Maps
}

\author{
Aileen R. Buckley \\ Esri, 380 New York Street, Redlands, CA, 92373m], USA, abuckley@esri.com
}

Keywords: Vietnam, topographic map, ArcGIS Pro

\begin{abstract}
:
The Vietnam War spanned nearly two decades, beginning in 1954 and ending with the withdrawal of the last U.S. forces in 1973. During the conflict, the U.S. Army Map Service (AMS) compiled, published, and distributed military topographic maps for use by U.S. military forces (NGA, 2021). Established in 1941, the AMS was re-designated the U.S Army Topographic Command Center (USATC) in 1968. It later merged into the Defense Mapping Agency-a heritage organization of the National Imagery and Mapping Agency (NIMA). To support the ground military units during the Vietnam War, the AMS/USATC, with the help of several U.S. Engineer Battalions, produced a series of topographic maps at a map scale of 1:50,000 for Vietnam and its immediately adjacent areas. Smaller scale maps at a scale of $1: 250,000$ were also produced.
\end{abstract}

Vietnam-era maps compiled to support U.S. military operations formed a "vital source of timely in-country knowledge at a critical time for American defense policy in Asia" (NGA, 2021). Through a cooperative agreement with the newly formed Republic of South Vietnam, American and Vietnamese cartographers and surveyors compiled a considerable knowledge base for the construction of comprehensive maps of the country. The resulting map collection "marked the first time that the American military mapping agencies had completed large- and medium-scale mapping of an area in advance of a major commitment of combat forces" (NGA and nga_geoint, 2021).

The 1:50,000-scale and 1:250,000-scale historical topographic maps for Vietnam, Cambodia, and Laos are now declassified. For this project, they were obtained from the National Archives at College Park, Maryland, scanned at a resolution of 600 dots per inch (DPI), and georeferenced in support of development of The National Map's Historical Topographic Map Collection (Then, 2012).

The 1:50,000-scale maps were produced from 1965 through 1971. The maps were in both English and Vietnamese and had a variant name of Series L7014 (for Vietnam) or L7016 (for border maps primarily covering a portion of Cambodia). The maps show relief with contours and spot heights in meters and bathymetric contours and depths in soundings. Vegetation, hydrography, cities and towns, cultural features, geographic place names, transportation networks, and other infrastructure were also noted on the maps. Marginalia includes notes, a glossary, an elevation guide diagram, a metric conversion graph, an administrative boundary diagram, and an index to adjoining sheets (Library of Congress, 2021).
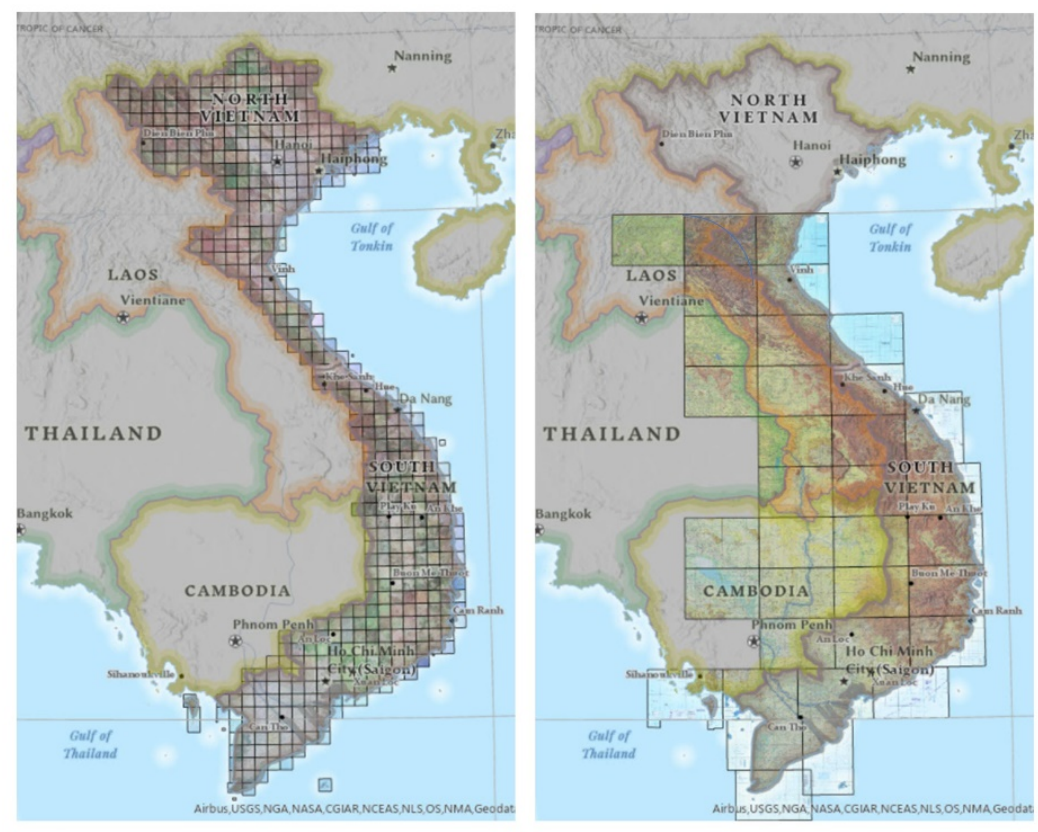

Figure 1. The 1:50,000-scale map collection (left); the 1:250,000-scale map collection (right). 
Using ArcGIS Pro, two map services - one for the 1:50,000-scale maps (Figure 1, left) and one for the 1:250,000 -scale maps (Figure 1, right) — were shared as hosted tile layers to ArcGIS Online. Hosted tile layers "support fast visualization of large datasets using a collection of pre-drawn map images or tiles" (Esri, 2021). An advantage of hosted tile layers is that they can be created without using ArcGIS Image Server, so users with ArcGIS Pro or Enterprise (or ArcMap) and an ArcGIS Online Organizational account can easily create them. The online map services can be used to: explore the maps in the collection, create online maps and apps, digitize or analyze the map contents in GIS, and download the maps in GeoTIFF format.

Each hosted tile layer was generated from a mosaic dataset - an ArcGIS geodatabase data model that allows multiple images (or rasters) to be managed as one catalog of rasters and viewed as a mosaicked image. A mosaic dataset has multiple contents, including, among others, a catalog that references the source of the images and footprints (extents) of each of the rasters and a feature class that defines the boundary around all the rasters. For the 1:50,000 scale Vietnam map collection, a second mosaic dataset was created for the insets that appeared on some of the maps in the collection (Figure 2, right). A third mosaic dataset was created with edited footprints for maps that had overedges (Figure 2, left). These two additional mosaic datasets were also shared as hosted tile layers in ArcGIS Online.
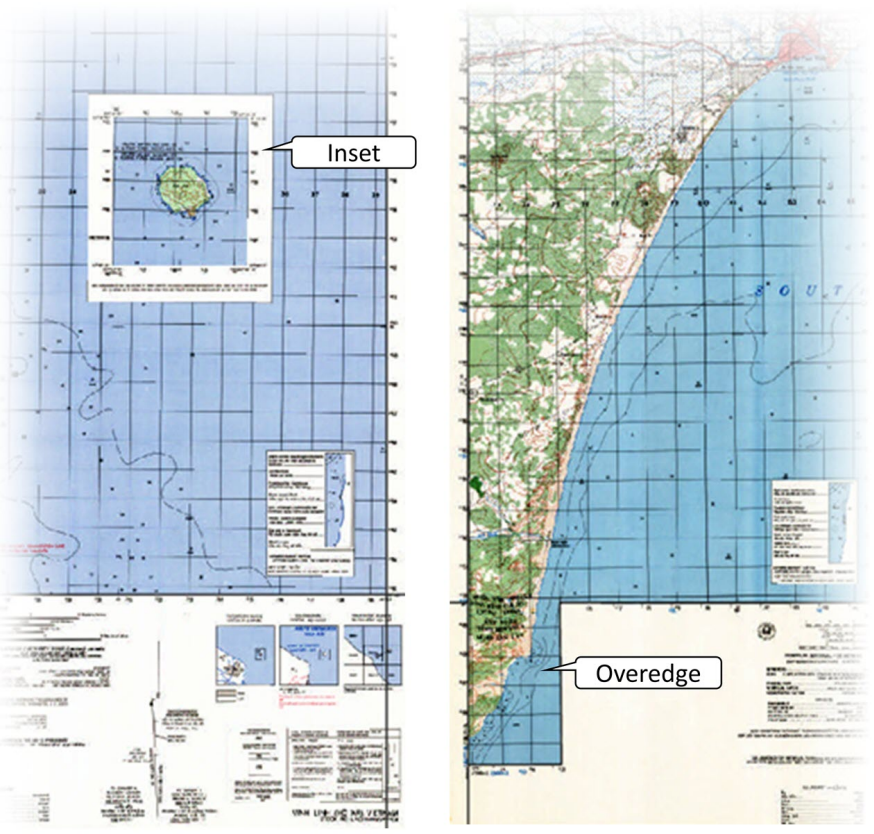

Figure 2. An example of an inset (left); an example of an overedge (right).

This project describes a unique collection of maps that have been shared online for all to access. It also demonstrates an easy and accessible workflow for ArcGIS that does not require the use of more complex parts of the platform-such as Image Server - so that other map collection managers can also put their content on the web and share it in a format that meets the varied needs of their patrons.

\section{References:}

Esri. 2021. "Publish hosted tile layers from files." ArcGIS Online Help | Documentation. Accessed June 20, 2021. https://doc.arcgis.com/en/arcgis-online/get-started/what-isagol.htm\#: :text=ArcGIS\%20Online \%20is\%20a\%20cloud,being\%20mobile\%20in\%20the\%20field.

Library of Congress. 2021. "Map: Vietnam 1:50,000.” LC Catalog - Item Information (Full Record). Accessed June 20, 2021.

https:/catalog.loc.gov/vwebv/search?searchCode=LCCN\&searchArg=2004630440\&searchType=1\&permalink=y.

National Geospatial-Intelligence Agency (NGA) and nga_geoint. 2021. "Pathfinder - Vol. 10, No. 1 (Jan/Feb 2012)." Issuu. Accessed June 20, 2021. https://issuu.com/nga_geoint/docs/2012_jan-feb/17.

National Geospatial-Intelligence Agency (NGA). 2021. "NGA in History - Defining Moments.” Accessed June 20, 2021. https://www.nga.mil/defining-moments/Army_Map_Service.html.

Then, Kenneth M.2021. Georeferencing and Archiving a Collection of Historical Topographic Maps of Vietnam”. Master's thesis (University of Wisconsin - Madison). 